\title{
Magnesium Sulfur Battery with a New Magnesium Powder Anode
}

\author{
B. Sievert ${ }^{\mathrm{a}}$, J. Häcker ${ }^{\mathrm{a}}$, F. Bienen ${ }^{\mathrm{a}}$, N. Wagner ${ }^{\mathrm{a}}$ and K.A. Friedrich ${ }^{\mathrm{a}, \mathrm{b}}$ \\ ${ }^{a}$ Institute of Engineering Thermodynamics, German Aerospace Center, Stuttgart, \\ Germany \\ ${ }^{\mathrm{b}}$ Institute for Energy Storage, University of Stuttgart, Germany \\ Herein we report a magnesium powder anode with medium \\ capacity retention and recovery of potential plateaus over \\ reversible cycling. Furthermore powder anodes showed an \\ optimized behavior during charging leading to an improved \\ coulomb efficiency compared to magnesium foil anodes. Battery \\ cells with a powder anode showed pronounced voltage plateaus \\ and no problem to reach a $2.8 \mathrm{~V}$ cut-off voltage. Within the \\ compaction pressure range investigated a magnesium powder \\ anode prepared by relatively low pressures showed advantageous \\ properties and was further investigated by electrochemical \\ impedance spectroscopy.
}

\section{Introduction}

Magnesium sulfur $(\mathrm{Mg} / \mathrm{S})$ secondary batteries present many advantages including a high theoretical capacity of sulfur $(1672 \mathrm{Ah} / \mathrm{kg})$ and magnesium $(2230 \mathrm{Ah} / \mathrm{kg}$ and $3832 \mathrm{Ah} / \mathrm{L})$, leading to a high theoretical energy density of a $\mathrm{Mg} / \mathrm{S}$ cell of $1600 \mathrm{Wh} / \mathrm{kg}$ gravimetrically and $3200 \mathrm{Wh} / \mathrm{L}$ volumetrically. Furthermore sulfur and magnesium are abundant and non-toxic materials. In contrast to lithium, magnesium is not susceptible to dendrite formation during deposition and can be the solution for a safe metal anode.

Unfortunately magnesium offers slower diffusion and reaction kinetics compared to lithium due to the divalent magnesium ion. Active material loss during cycling similar to lithium sulfur batteries can be observed, likely due to polysulfide shuttle mechanism (1). Common electrolyte salts form a passivation layer on the magnesium anode (SEI) which is non-permeable for magnesium ions $(2,3)$. The development of components and processes applicable to large-scale production and low cost fabrication is also a great challenge for the commercialization of metal sulfur batteries. Due to the low electrical conductivity of sulfur, the incorporation of a conductive material in the cathode is one of the main issues related to the fabrication of the cathode (4). A suitable electrolyte for $\mathrm{Mg} / \mathrm{S}$ has to be non-nucleophilic, which is the prerequisite for stability towards both electrodes, may not form a blocking layer and offer sufficient ionic conductivity. Before the introduction of the first non-nucleophilic electrolytes no functional secondary $\mathrm{Mg} / \mathrm{S}$ battery could be demonstrated. Only few works about $\mathrm{Mg} / \mathrm{S}$ systems have been published (1), (5-9).

\section{Non-nucleophilic Electrolyte}

In 2011 the first battery system with a magnesium anode and a sulfur cathode was demonstrated by Kim et.al. (7). As mentioned the development of the electrolyte 
represented a particular challenge, since it should not form inhibiting layers on the magnesium or react with the sulfur of the cathode. Kim et. al. used nucleophilic magnesium organohaloaluminate that caused a rapid breakdown of the sulfur cathode due to the electrophilic nature of sulfur and immediate formation of sulfide reaction products. By combining a non-nucleophilic organomagnesium halide (hexamethyldisilazanemagnesium chloride, $\mathrm{HMDSMgCl}$ ) and a Lewis acid (aluminum chloride) Liebenow et al. demonstrated reversible magnesium stripping and plating. (10).

A simplified preparation of the HMDSMgCl-electrolyte by mixing a bisamide with aluminum chloride was reported by Zhao-Karger et. al. without crystallization-steps (11) and the addition of magnesium chloride (5).

\section{$\underline{\text { Sulfur Carbon Cathode }}$}

The development of components and processes applicable to large-scale production at low cost is a great challenge for the commercialization of metal-sulfur-batteries (4). Identifying a cheap carbon structure of low weight, homogeneous structure and good electrochemical performance is significant (12). The use of carbon materials was intensively investigated in terms of lithium sulfur (LiS) battery development reviewed in detail in (13). The use of CMK-3, a homogeneously structured mesoporous carbon (14) or rGO, a reduced graphene oxide (15), for example are frequently reported as suitable conductive matrices based on the good cycling performance in LiS batteries. Both cathode conductive matrices have been also evaluated in $\mathrm{Mg} / \mathrm{S}$ system(5, 6). For both carbon materials the price is at least $100 \$ / \mathrm{g}$ which makes them interesting as a model system rather than a feasible raw material for large scale applications. The use of commercially available conductive carbon blacks in the price range of 0.01-0.03 \$/g and the mechanical intrusion of sulfur are scalable approaches that do not produce waste (13). The use of activated carbon fiber (ACF) has been tested with remarkably good results (1) as well as activated carbon cloth (ACC) (8). Even though the mentioned cathode structures are able to stabilize cycling performance to some degree, further enhancements were achieved by the optimization of electrolytes or the development of specialized functional layers $(1,8)$.

It should be mentioned that corrosion was reported for the aluminum substrate in $\mathrm{Cl}^{-}$ containing magnesium electrolyte (9) as it was used for in this work. Carbon coating was shown to efficiently protect the Al-foil against corrosion (16). But in most cases the use of Inconel 625 as substrate $(5,6)$ or current collector rods (8) in Swagelok type cells is reported.

\section{Magnesium Anode}

Compared to studies on appropriate electrolytes and sulfur-cathodes, magnesium anodes have received little attention in the $\mathrm{Mg} / \mathrm{S}$ system. So far, mainly magnesium foil has been used as anode in $\mathrm{Mg} / \mathrm{S}$ battery studies $(1,7,8)$. Magnesium powder anodes have been used twice but their preparation and the influence on the behavior of the cell have received little attention $(5,6)$. Such powdered anodes were made by isostatically compressing ball milled magnesium powder and conductive carbon black to form pellets. Graphite has not been used for such anodes yet. The intercalation of magnesium ions into graphite was reported to be irreversible (17). Nevertheless, graphite is considered a 
promising candidate for powdered anodes due to its high electronic conductivity and superior percolation properties.

Since side reactions with electrolyte decomposition play an important role in Metal-S batteries and contribute to rapid degradation of $\mathrm{Mg} / \mathrm{S}$ cells. A symmetrical magnesium cell is a valuable attempt to distinguish anode and cathode performance (18). Finding the appropriate combination of cathode, anode and electrolyte chemistry is the most important challenge of developing a rechargeable battery. However, it is imperative also to examine the individual components in order to understand the interactions in the cell.

\section{$\underline{\mathrm{Mg} / \mathrm{S} \text { Cell Performance }}$}

$\mathrm{Mg} / \mathrm{S}$ cells performance challenges are numerous: Reaching a high cut-off-voltage of $2.8 \mathrm{~V}$ during charging is often reported to be a problem $(5,7,8)$. For this reasons cut-offvoltage during charging of $2.5 \mathrm{~V}$ and $2.4 \mathrm{~V}$ are commonly used (6). Until now only cells equipped with ACF cathodes reached cut-off-voltages of $2.8 \mathrm{~V}$ and $3.0 \mathrm{~V}$ during charging (1). Often, the characteristic voltage plateaus during discharge are already absent after the first cycle with an associated fast capacity fade $(1,6,7)$. The absence of the voltage plateaus and the fast loss of capacity after the first cycle was claimed to indicate irreversibility of the $\mathrm{Mg} / \mathrm{S}$ cell, while the addition of $\mathrm{Li}$ ion into the electrolyte could enhanced reversibility (8). This view, however, is inconsistent with a report by $\mathrm{Yu}$ and Manthiram with superior reversible capacities (1). Undesired and still unclear side reactions during charging may in our opinion be responsible for the inconsistent results. Herein we report the preparation and performance of a $\mathrm{Mg}-\mathrm{C}$ anode with improved recovery of potential plateaus during reversible cycling in comparison to other $\mathrm{Mg}-\mathrm{C}$ anodes and compared to magnesium foil in $\mathrm{Mg} / \mathrm{S}$ cells.

\section{Experimental}

\section{Materials synthesis}

Synthesis of the Sulfur Cathodes. Sulfur powder (Alfa Aesar, sublimed, 100 mesh, 99.5\%) and conductive carbon black (TIMCAL C-NERGY SUPER C65) were each dispersed via ball milling in a planetary mill (Retsch, PM400) and zircon oxide balls with a diameter of $5 \mathrm{~mm}$ in dimethyl sulfoxide (DMSO, VWR Chemicals, dried, water content below $0.03 \%$ ). Polyvinylidene fluoride binder (Solvay, Solef 5130) was dissolved in DMSO separately. The as prepared solution and suspension were mixed in two different relations according to the solid content fractions listed in Table I. The $50 \mathrm{wt} . \mathrm{\%}$ and $70 \mathrm{wt} .-\%$ sulfur cathodes are named $50 \mathrm{~S}$ cathode and $70 \mathrm{~S}$ cathode in the following.

TABLE I. Summary of cathode composition

\begin{tabular}{ccc}
\hline Ingredient & $\begin{array}{c}\text { 70S cathode } \\
\text { w / wt. } \%\end{array}$ & $\begin{array}{c}\text { 50S cathode } \\
\text { w / wt. } \%\end{array}$ \\
\hline sulfur (active material) & 70 & 50 \\
Super C65 & 20 & 40 \\
(carbon black, conductive matrix) & & 10 \\
Solef 5130 & 10 & \\
(Polyvinylidenfluoride, binder) & & \\
\hline
\end{tabular}


The mixtures were applied to carbon coated aluminum via the doctor blade coating method and dried for $24 \mathrm{~h}$ at $60^{\circ} \mathrm{C}$ followed by a vacuum drying step for $1 \mathrm{~h}$. The punched cathodes had a diameter of $18 \mathrm{~mm}$ and a sulfur loading of $1 \mathrm{mg} / \mathrm{cm}^{2}$.

Synthesis of the Metal Powder Anodes. As received magnesium powder (Alfa Aesar, 325 mesh, 99.8\%) and dried graphite (TIMCAL Graphite C-NERGY KS6L) were ball milled together in the weight ratio 80:20 using a roller mixer (Ratek BTR5) with an $250 \mathrm{ml}$ polypropylene vial and zircon oxide balls with a diameter of $10 \mathrm{~mm}$ under an argon atmosphere at a moderate speed of $30 \mathrm{rpm}$ for $5 \mathrm{~h}$. The grinded powder was pressed with a hydraulic press and a homemade die with a diameter of $18 \mathrm{~mm}$ with variant pressure of $75 \mathrm{MPa}$ and $350 \mathrm{MPa}$. Within this pressure variation sufficiently stable anodes were prepared which we named powder anode at low pressure (PALP) and powder anode at high pressure (PAHP).

For evaluating magnesium powder anode performance, cells with magnesium foil anode (Goodfellow, $250 \mu \mathrm{m}$ thickness, 99.9\%) were used as references according to the above mentioned procedure. Magnesium foil anode surface was intensively polished under argon before usage to avoid magnesium oxide surface layer.

For EIS measurements of symmetrical magnesium cells the above mentioned procedures were applied with two similar magnesium anodes.

Synthesis of Non-nucleophilic Magnesium Electrolyte. The electrolyte was synthesized as reported in literature (5). All reactants were dried before use. All syntheses were performed in an argon filled glove box. In a batch synthesis, $1.13 \mathrm{~g}$ of bishexamethyldisilazide magnesium ((HMDS) $\left.{ }_{2} \mathrm{Mg}\right)$, Sigma Aldrich, 97\%) was dissolved in $4 \mathrm{~mL}$ of diethylene glycol dimethyl ether (DEG), Sigma Aldrich). Then $0.96 \mathrm{~g}$ of aluminum chloride $\left(\mathrm{AlCl}_{3}\right.$, Sigma Aldrich, 99,99\%) was slowly added and stirred for $24 \mathrm{~h}$. Afterwards, $0.34 \mathrm{~g}$ of magnesium chloride $\left(\mathrm{MgCl}_{2}\right.$, Sigma Aldrich, $\left.99.99 \%\right)$ was added into the mixture and further stirred for $40 \mathrm{~h}$. Afterwards the mixture was diluted with $2 \mathrm{~mL}$ of ionic liquid (IL) N-methyl-N-butylpiperidinium bis(trifluoromethanesulfonyl) imide (PP14TFSI, Iolitec). $\mathrm{Mg}$ ion concentration was $1.2 \mathrm{M}$ after dilution and the resulting electrolyte will be named 1.2 M DEGIL in the following. In a similar attempt $1.5 \mathrm{MMg}$ ion electrolyte was prepared in a $1: 1$ by volume mixture of DEG and tetraethylene glycol dimethyl ether (TEG) named 1.5 M DEGTEG.

\section{$\underline{\text { Methods }}$}

Cell Preparation. Electrochemical measurements were conducted in Swagelok type cell, described elsewhere (19). The current collectors were protected from corrosion with carbon-coated aluminum sheets. The cell comprised a sulfur cathode electrode, magnesium powder anode electrode, glass fiber separator (Whatman GF/C) and $150 \mu \mathrm{L}$ non-nucleophilic electrolyte. Both electrodes had a diameter of $18 \mathrm{~mm}$, while the separator was $22 \mathrm{~mm}$. Assembling of the cells was conducted in an argon filled glove box (GS GLOVEBOX) with water and oxygen content below 3 ppm.

Cycling Performance. A Basytec CTS test station with corresponding Basytec software was used to carry out the electrochemical testing of the batteries. After assembling the cell the open circuit voltage was measured for a period of $1 \mathrm{~h}$. Then the charge-discharge procedure was performed galvanostatically at a current density of $167 \mathrm{~A} / \mathrm{kg}_{\text {Sulfur }}$ in a voltage range of $2.8 \mathrm{~V}-0.5 \mathrm{~V}$. After reaching the end-of-charge 
voltage of $2.8 \mathrm{~V}$, a potentiostatic period followed until the current either declined to $10 \%$ of its original value or, for at least $15 \mathrm{~min}$ before the next cycle began.

If the cell was unable to reach cut-off voltages of $2.8 \mathrm{~V}$, the cut-off voltage was reduced to $2.5 \mathrm{~V}$ with a prolonged potentiostatic period.

Electrochemical Impedance Spectroscopy (EIS) of Symmetrical Magnesium Cells. Zahner ${ }^{\circledR}$ IM6 with the Thales battery software was used to carry out the impedance measurement of symmetrical magnesium cells. A frequency range from $1 \mathrm{MHz}$ to $100 \mathrm{mHz}$ and an excitation voltage of $5 \mathrm{mV}$ were applied for performing the EIS measurements.

\section{Results and Discussion}

\section{$\underline{\text { Sulfur Cathode Performance. }}$}

In figure 1 charge and discharge capacity (1a, 1b) and coulombic efficiency (1c) as a function of cycle number and corresponding voltage profiles (1d-1f) as a function of capacity are shown. The values were obtained with a 50S cathode and $70 \mathrm{~S}$ cathode versus a PALP electrode in 1.2 MDEGIL named 50S/DEGIL/PALP and 70S/DEGIL/PALP. The composition of the cathodes is described in table I and both types of cathodes were used with the same sulfur loading of $1 \mathrm{mg} / \mathrm{cm}^{2}$. The cathodes differ in their solid component composition. While the binder content remains constant at $10 \mathrm{wt} . \%$, the sulfur content increases from $50 \mathrm{wt} . \%(50 \mathrm{~S})$ to $70 \mathrm{wt} . \%(70 \mathrm{~S})$, which is compensated by lowering conductive carbon black fraction from $40 \mathrm{wt} . \%$ (50S) to $20 \mathrm{wt} . \%$ (70S). $50 \mathrm{~S} / \mathrm{DEGIL} / \mathrm{PALP}$ had a discharge capacity of about $600 \mathrm{Ah} / \mathrm{kg}_{\text {Sulfur }}$ in the first cycle while $70 \mathrm{~S} / \mathrm{DEGIL} / \mathrm{PALP}$ was $570 \mathrm{Ah} / \mathrm{kg}_{\text {Sulfur. }}$. Until the third cycle the difference in capacity value increased to $90 \mathrm{Ah} / \mathrm{kg}_{\text {Sulfur. In the subsequent cycles this value decreased }}$ until it reached a steady value of about $30 \mathrm{Ah} / \mathrm{kg}_{\text {Sulfur }}$ after the tenth cycle. The coulomb efficiency also reached a constant value of 0.8 till 0.9 after the first ten cycles as shown in figure 1c. In comparison to 50S/DEGIL/PALP, 70S/DEGIL/PALP showed slightly lower coulombic efficiency over cycling and higher overpotential between discharge and charge profile.

The overpotential is illustrated exemplary in figure 2 , where the voltage versus relative capacity is plotted for discharge and charge. The overpotential is caused by the higher sulfur fraction in 70S cathode since all other parameters such as type and amount of electrolyte, anodes and areal sulfur loading have been kept constant. For higher sulfur loading the higher overpotentials are expected due to the low electronic conductivity of sulfur and the discharge sulfur products. For HMDSMgCl electrolyte coulomb efficiency and cycle life is the highest reported in literature. However, the influence of the different cathodes on cycle stability is minor. The next paragraph investigates the behavior of different magnesium anodes in the studied system. 

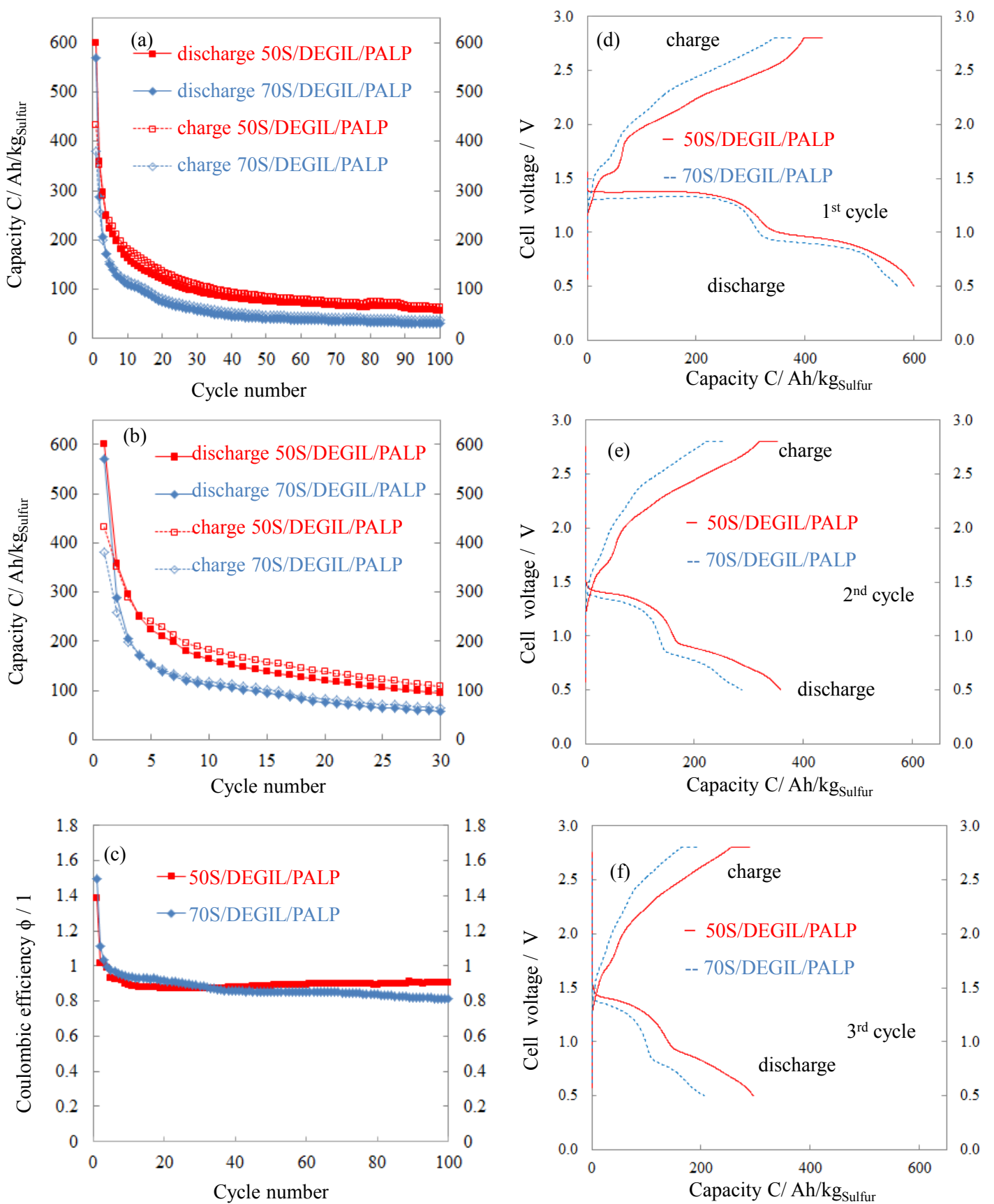

Figure 1. Discharge and charge capacity ( $a, b$ (cutout)) and coulomb efficiency (c) as a function of cycle number of 50S/DEGIL/PALP and 70S/DEGIL/PALP; corresponding discharge-charge profiles (d-f) of the same cells. 


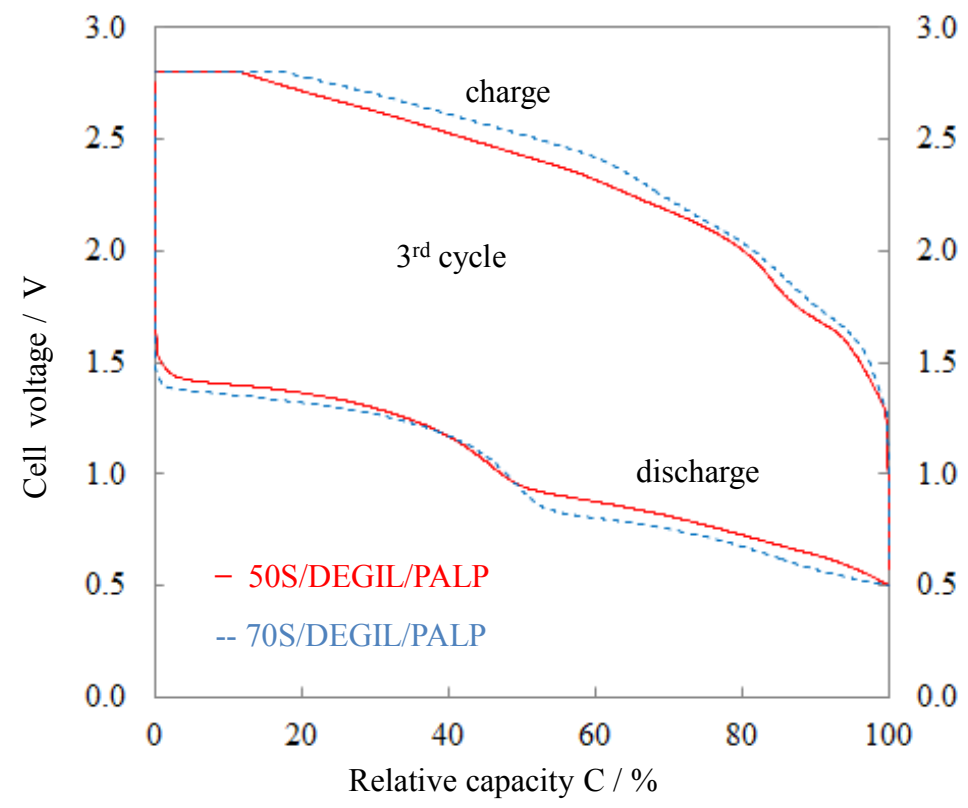

Figure 2. Discharge-charge profile versus relative capacity of 50S/DEGIL/PALP and 70S/DEGIL/PALP.

\section{Magnesium Anode Performance.}

Magnesium anode behavior was compared in $\mathrm{Mg} / \mathrm{S}$ cells comprising a $50 \mathrm{~S}$ cathode, 1.2 M DEGIL electrolyte and either a low pressure die pressed (PALP) or high pressure die pressed (PAHP) powder anode. The PALP was visually dull and able to soak up electrolyte, while the PAHP had a shiny surface and no tendency to soak up electrolyte. PAHP appeared to be similar to a dense magnesium foil. To make a direct comparison, magnesium foil was included into the cell testing for classifying the magnesium powder anode performance. Interestingly it was not possible for us to build a working magnesium foil cell with 1.2 M DEGIL but only with a 1.5 M DEGTEG, comprising TEG instead of an ionic liquid as secondary solvent. With 1.5 MDEGTEG the voltage profile of the tested cells changed as shown in figure 3. Only the first voltage plateau occurred with PALP electrode in 1.5 MDEGTEG and it was problematic to reach the defined end-ofcharge cut-off voltage consistent with literature $(5,7,8)$. Therefore the cut-off voltage during charge of $50 \mathrm{~S} / \mathrm{DEGTEG} / \mathrm{Mg}$ foil cell was reduced to $2.5 \mathrm{~V}$. Even with the modified cycling conditions it was not possible to obtain more than 15 cycles with this cell set up as it is shown in figure 4c. 


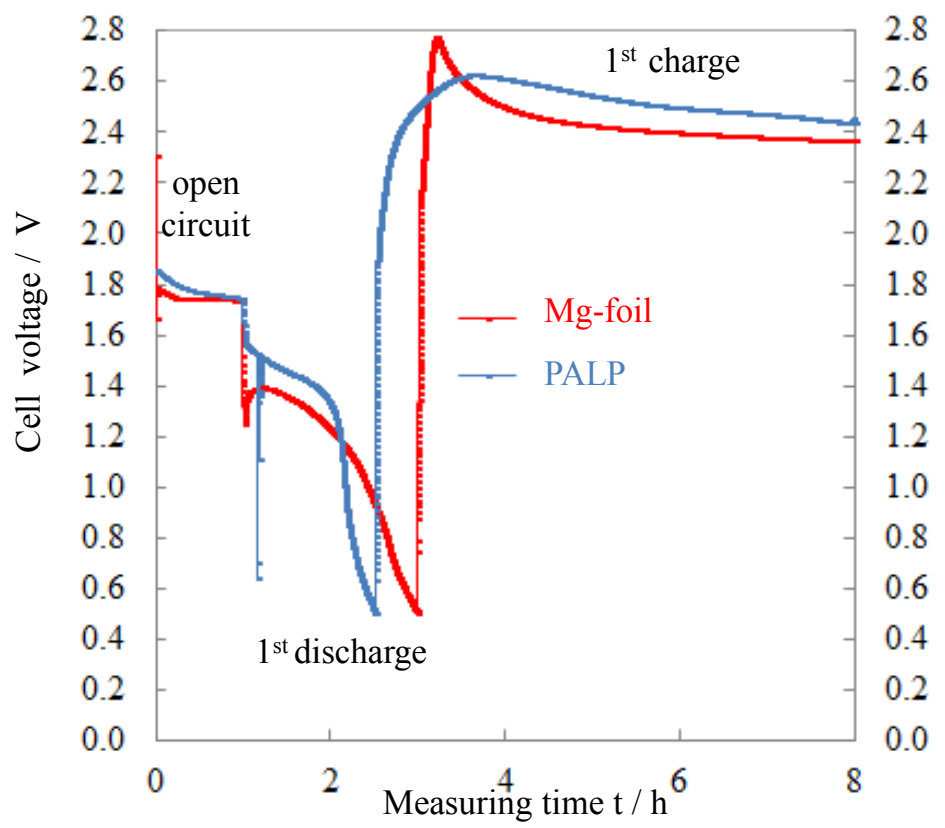

Figure 3. Discharge-charge profiles of magnesium foil and PALP in 1.5 M DEGTEG.

In figure 4 various discharge-charge voltage profiles of 50S/DEGIL/PALP cell (4a), 50S/DEGIL/PAHP cell (4b) and 50S/DEGTEG/Mg foil cell (4c) were compared. The most pronounced and best recovered voltage plateaus could be obtained with PALP electrode in 50S/DEGIL/PAHP cell as was already shown in figure 1 . In contrast, with a PAHP electrode (figure 4b) already the first cycle discharge performance was inferior. In the subsequent cycles the discharge profile was raised somewhat but only one plateau occurred and diminished immediately until it disappeared in the fifth cycle. In the tenth cycle a lower plateau could be observed at about $0.7 \mathrm{~V}$. From the tenth cycle forward the capacity retention of the cell with PALP and PAHP is almost the same. The discharge voltage profiles of $50 \mathrm{~S} / \mathrm{DEGTEG} / \mathrm{Mg}$ foil (figure $4 \mathrm{c}$ ) were similar to that of 50S/DEGIL/PAHP, except for the dramatic drop of the first discharge and the more pronounced lower plateau. While a similar discharge voltage profile could be observed charging was strongly inhibited with the magnesium foil. We tried to reduce the constant voltage period to achieve a reduced amount of charge, comparable to the previously obtained charge quantity during discharging, but this resulted in less achievable discharge capacity and was not further applied. Due to the unexpected behavior of magnesium foil during charging in DEGIL, which, however, shows adequate behavior with powder anodes according to figure 4, impedance spectroscopy was applied for clarification in symmetrical magnesium cells. 

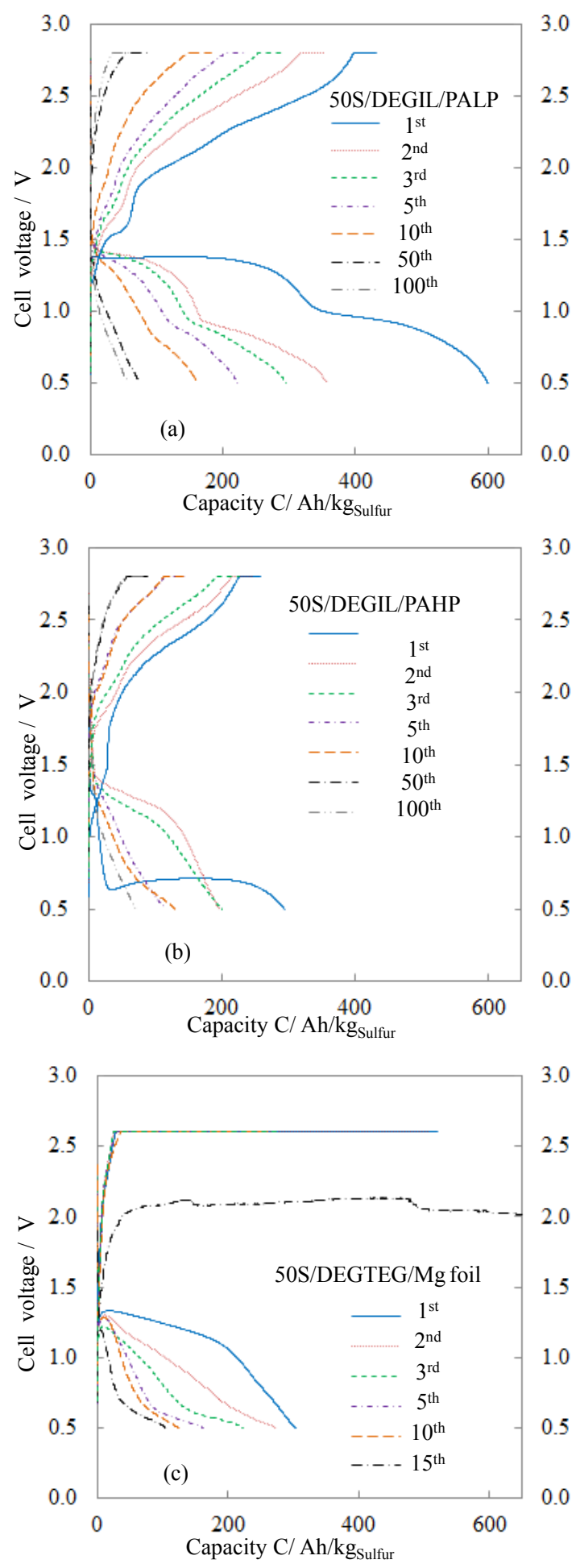

Figure 4. Discharge-charge profiles of PALP, PAHP and magnesium foil anodes with $50 \mathrm{~S}$ cathode.

In figure 5 Bode (figure 5a) and Nyquist representations (figure 5b) of the three different types of magnesium anodes were compared in DEGTEG electrolyte. DEGTEG 
was chosen because all three electrode types were operational in this electrolyte, compared to the Mg foil anode in DEGIL. The influence of the electrolyte properties, for example its ionic conductivity and viscosity will be a topic of further investigation. In the high frequency region at a phase angle of zero degree a resistance $\mathrm{R}$ can be determined as shown in figure $5 \mathrm{a}$. $\mathrm{R}$ represents the ohmic resistance contribution resulting from the electrolyte resistance, inner electrode resistance, current collector contact resistance and cell connections. Changes observed in ohmic resistance are normally associated with variations in electrolyte properties such as chemical composition or degradation. In this setup an influence of the different anodes itself must be considered. For $\mathrm{Mg}$ foil (I) and PAHP (II) anode an ohmic resistance of $\mathrm{R}_{\mathrm{I}, \mathrm{II}}=8 \Omega$ was determined. With less compressed PALP (III) anode an ohmic resistance of $\mathrm{R}_{\mathrm{III}}=19 \Omega$ was observed. All three cells comprised of the same electrolyte and-amount. Due to the porous nature of PALP electrode electrolyte was soaked into the bulk electrode. This results in impedance due to the pore electrolyte. The phase angle below $-45^{\circ}$ of the PALP/DEGTEG/PALP spectra is an evidence of the porous nature of PALP electrode. Porous electrodes show a low phase angle of $-45^{\circ}$ while dense electrodes show a phase angle of $-90^{\circ}$ (20). In order to emphasize the characteristics of the porous electrode in comparison to the dense powder anode and the metal foil, the results of the impedance measurement were additionally shown Nyquist representation. In a future work the measurement will be investigated with porous electrode equivalent circuit model. 

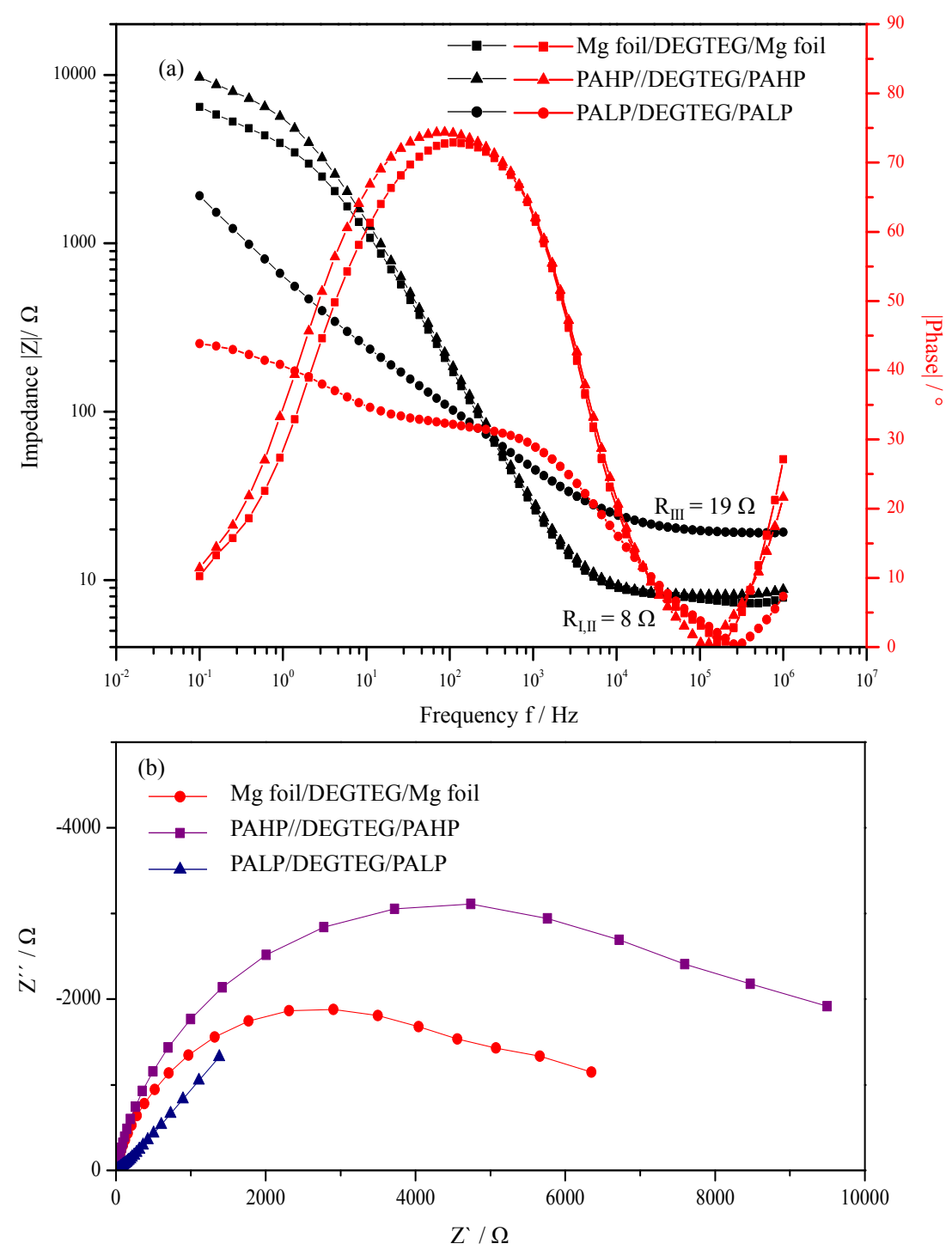

Figure 5: Bode (a) and Nyquist plot (b) for uncycled symmetrical magnesium cells at room temperature with Mg foil, PAHP and PALP anodes with DEGTEG electrolyte.

\section{Conclusion}

The proof of concept of a working porous magnesium powder anode comprising of graphite and magnesium powder has been shown. Such porous electrodes showed improved cycling behavior compared to dense powder and magnesium foil electrodes. Magnesium powder anode showed medium capacity retention and recovery of potential plateaus over reversible cycling. Powder anodes showed an optimized behavior during charging leading to an improved coulombic efficiency compared to magnesium foil anodes. The porous nature of powder anodes prepared with lower compression (PALP) was indicated by electrochemical impedance spectroscopy. Future work will investigate the effect of electrolyte properties as well as in depth-impedance analysis of the electrodes and cells. 


\section{Acknowledgments}

We thank the Federal Ministry of Education and Research for funding this work.

FKZ: 03 XP0032A

\section{References}

1. 1. X. Yu and A. Manthiram, ACS Energy Lett., 1(2), 431, (2016).

2. Z. Lu, A. Schechter, M. Moshkovich, and D. Aurbach, J. Electroanal. Chem., 466, 203, (1999).

3. R. Mohtadi and F. Mizuno, Beilstein J. Nanotechnol., 5, 1291, (2014).

4. N. A. Cañas, A. L. P. Baltazar, M. A. P. Morais, T. O. Freitag, N. Wagner, and K. A. Friedrich, Electrochim. Acta, 157, 351, (2015).

5. Z. Zhao-Karger, X. Zhao, D. Wang, T. Diemant, R. J. Behm, and M. Fichtner, Adv. Energy Mater., 5(3), 1401155 (2015).

6. B. P. Vinayan, Z. Zhao-Karger, T. Diemant, V. S. K. Chakravadhanula, N. I. Schwarzburger, M. A. Cambaz, R. J. Behm, C. Kübel, and M. Fichtner, Nanoscale, 8(6), 3296, (2016).

7. J. Muldoon, H. S. Kim, T. S. Arthur, G. D. Allred, J. Zajicek, J. G. Newman, A. E. Rodnyansky, A. G. Oliver, and W. C. Boggess, Nat. Commun., 2, 427, (2011).

8. T. Gao, M. Noked, A. J. Pearse, E. Gillette, X. Fan, Y. Zhu, C. Luo, L. Suo, M. A. Schroeder, K. Xu, S. B. Lee, G. W. Rubloff, and C. Wang, J. Am. Chem. Soc., 137(38) 12388, (2015).

9. Z. Zhao-Karger, M. Elisa, G. Bardaji, O. Fuhr and M. Fichtner, J. Mater. Chem. A, 5, 10815 (2017).

10. C. Liebenow, Z. Yang, P. Lobitz, and E. Moritz, Electrochem. Commun., 2, 641, (2000).

11. Z. Zhao-Karger, X. Zhao, O. Fuhr and J. M. Fichtner, RSC Advances 3, 16330, (2013).

12. M. Hagen, S. Dörfler, P. Fanz, T. Berger, R. Speck, J. Tübke, H. Althues, M. J. Hoffmann, C. Scherr, and S. Kaskel, J. Power Sources, 224, 260, (2013).

13. B. Lars, M. Oschatz, and S. Kaskel, Chem. Eur. J. 22. 1, (2016).

14. X. Ji, K. T. Lee and L. F. Nazar, Nat. Mater., 8(6), 500 (2009).

15. S. Evers and L. F. Nazar, Chem. Commun., 48, 1233 (2012).

16. C. Wall, Z. Zhao-Karger, and M. Fichtner, ECS Electrochem. Lett., 4(1), C8, (2014).

17. P. Novák, R. Imhof, and O. Haas, Electrochimica Acta 45, 351, (1999).

18. O. Tutusaus, R. Mohtadi, N. Singh, T. S. Arthur, and F. Mizuno, ACS Energy Lett.,2 224, (2016).

19. N. A. Cañas, K. Hirose, B. Pascucci, N. Wagner, K. A. Friedrich, and R. Hiesgen, Electrochim. Acta, 97, 42, (2013).

20. N. Wagner in Electrochemical Power Sources - Fuel cells, Evgenij Barsoukov and J. Ross Macdonald, Editors, Impedance Spectroscopy: Theory, Experiment, and Applications, 2nd Edition, John Wiley\&Sons p. 497, (2005). 